\title{
Reactors for Electric Furnace Circuits
}

\author{
BY HARRY A. WINNE \\ Power \& Mining Engineering Dept., General Electric Co., Schenectady, N. Y.
}

$\mathbf{I}$ $\mathrm{N}$ any alternating-current circuit supplying power to an electric arc furnace, the reactance of the circuit is depended upon to stabilize the arc, and to limit the maximum current which will flow through the circuit in case the arc becomes short-circuited, due to the electrodes coming into direct contact with each other or with the furnace charge. The resistance of the buses, electrodes and charge will help to limit the maximum current, but its effect is small in comparison with that of the reactance.

It is the purpose of this paper to discuss briefly the various methods of introducing reactance into furnace circuits, but not to attempt to determine what the amount of this reactance should be. This value will vary widely in different installations, and depends upon the limit which must be placed on the maximum current surges in the circuit, always taking into consideration the fact that as the reactance is increased the power factor of the load will decrease.

As a matter of interest is included the curve in Fig. 1 illustrating graphically the relation between reactance and power factor in a circuit. As abscissas are plotted reactance voltages in percentage of the supply voltage, while the ordinates represent the power factor of the circuit in per cent. It might be noted that maximum current can be limited to twice normal value, by using 50 per cent reactance, with a normal load power factor of 86.6 per cent, which is higher than the overall power factor of the load drawn by the average induction motor-driven manufacturing plant. A power factor as high as 95 per cent at normal load can be obtained with an amount of reactance which will limit the maximum current to only slightly more than three times normal.

Incidentally, the ordinates of this curve also indicate the voltage drop due to resistance in the circuit, or, neglecting the resistance in the transformer and furnace leads, the actual voltage which is obtained across the furnace electrodes in per cent of the supply voltage.

In the following discussion the electrical circuit of the installation, or briefly the furnace circuit, is considered as beginning at the circuit breaker which connects the furnace transformer to the power supply line. The total reactance of this circuit is made up of several components namely the reactance of the transformer, of reactance coils if such are used, of the heavy current leads from the transformer to the furnace, including the electrodes, and of the charge itself.

The reactance of the transformer can be varied within limits, as can that of the leads and buses by changing the spacing between and the arrangement of

"Presented at the Boston Meeting of the A.I. E. E., A pril 9, 1920 the conductors, but practically there is a minimum value for any given installation below which the reactance cannot be diminished.

This minimum limit increases with increasing currentcarrying capacity of the installation, and may actually become a serious handicap on very large furnaces.

However, there are numerous cases in which it is desirable to have a reactance even higher than what may be termed the "normal" value for the circuit. Such conditions may arise with furnaces of small capacity, on steel or other metal melting furnaces in which a high voltage is used for melting and a lower for refining, or when it is necessary to limit the maximum current surges to a very low value. In such instances it becomes necessary to adopt some means for inserting reactance into the circuit.

The means available for this purpose are: to make the transformer of high reactance; to so arrange the heavy current conductors as to obtain the desired effect; or to use external reactance coils on either the high-voltage or low-voltage side of the transformer.

\section{High-REACTANCE TRANSFORMERS}

The ordinary power transformer has an inherent reactance of from 2 per cent to 6 per cent. This value can usually be increased to a maximum of about 20 per cent without excessively increasing the cost or decreasing the efficiency of the transformer. Consequently, if a reactance of not over 14 per cent to 18 per cent in addition to the normal value is desired permanently in the circuit, the transformer manufacturer can usually incorporate it in the transformer.

This method of obtaining additional reactance is only satisfactory if the high reactance is desired under all conditions of furnace operation, and should not be used when it is advantageous to vary the reactance during the operating cycle. Neither is it ordinarily satisfactory on melting furnace installations using different voltages during different portions of the operating cycle, as with the usual system of tapping the transformer highvoltage winding to obtain variable low voltages, the percentage reactance of the transformer is higher for the low-voltage connection than for the high. That is, a transformer designed to give voltages of 120 and 80 , and have a reactance of say 10 per cent on the 120 -volt connection, might have a reactance of 15 per cent on the 80 -volt connection. The effect of this circumstance is decidedly disadvantageous, as the percentage reactance of the buses and cables is greater at the lower voltage,-assuming the same current,-than at the higher voltage, whereas a lower total reactance at the lower voltage would be advantageous. 
Additional Reactance Obtained by ARrangement OF CONDUCTORS

Another method of obtaining additional reactance, when the same is desired in the circuit continuously, is to arrange the heavy current leads from the transformer to the furnace with a comparatively wide space between those of different phases.

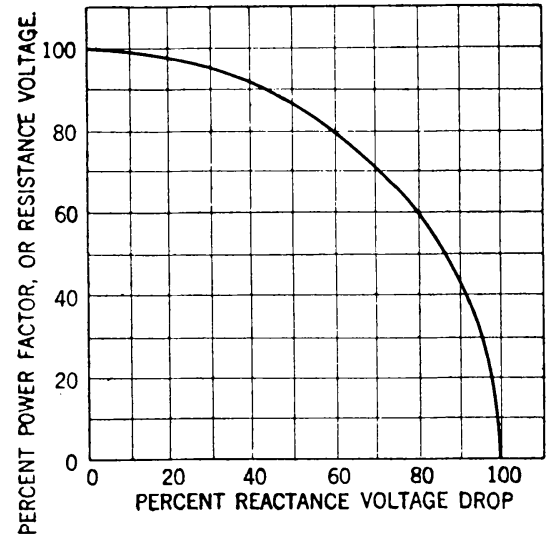

Fig. 1-Relation Between Reactance and Power Factor of a Circuit at Normal Kv-a. Load

Where the current per phase is high and the frequency 60 cycles, a considerable amount of reactance can be obtained in this way. As a matter of fact on furnaces of large capacity it is usually necessary to place the leads very close together, and sometimes interlace them, in order to prevent too high a reactance in the circuit.

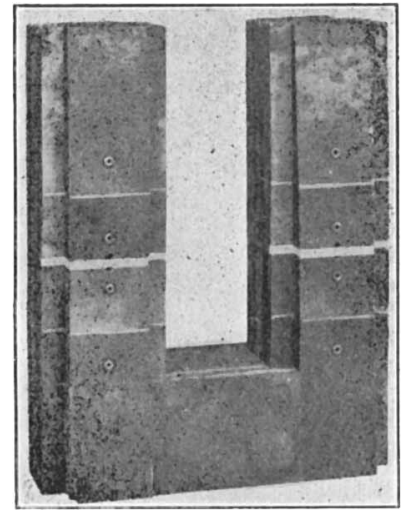

Fig. 2-Core of Iron-Core Reactor Light Shaded Sections Represent Air Gap

As a consequence it is usually only on smaller furnaces, or furnaces using a high voltage during a portion of their cycle, that special provision for obtaining high reactance is necessary.

\section{EXTERNAL-REACTANCE CoILs}

In many electric furnaces melting and refining steel and other metals a higher voltage is used during the melting than during the refining period. Owing to the higher voltage, and to the fact that conditions in the furnace during the melting portion of the cycle are such as to cause frequent current surges, reactance coils are often inserted in the circuit while the higher voltage is being used. These coils may be of either the iron-core or air-core type, and may be connected either in the

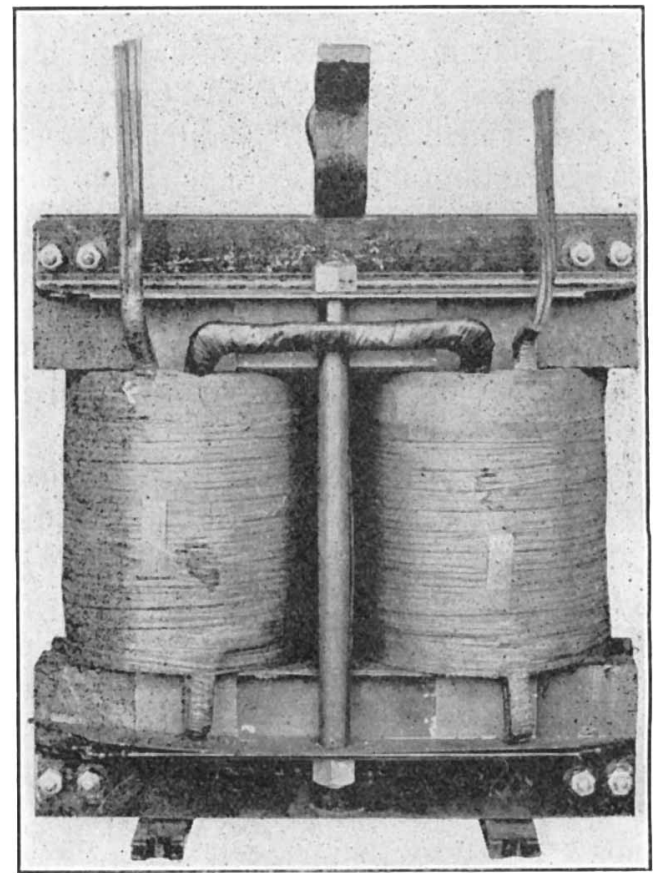

Fig. 3-Iron-Core Reactor Ready for Assembly in Tank

high-tension supply circuit to the transformer, or between the transformer and the furnace.

\section{IRON-CORE REACTORS}

The iron-core reactor consists simply of a number of turns of insulated conductor, wound upon a laminated

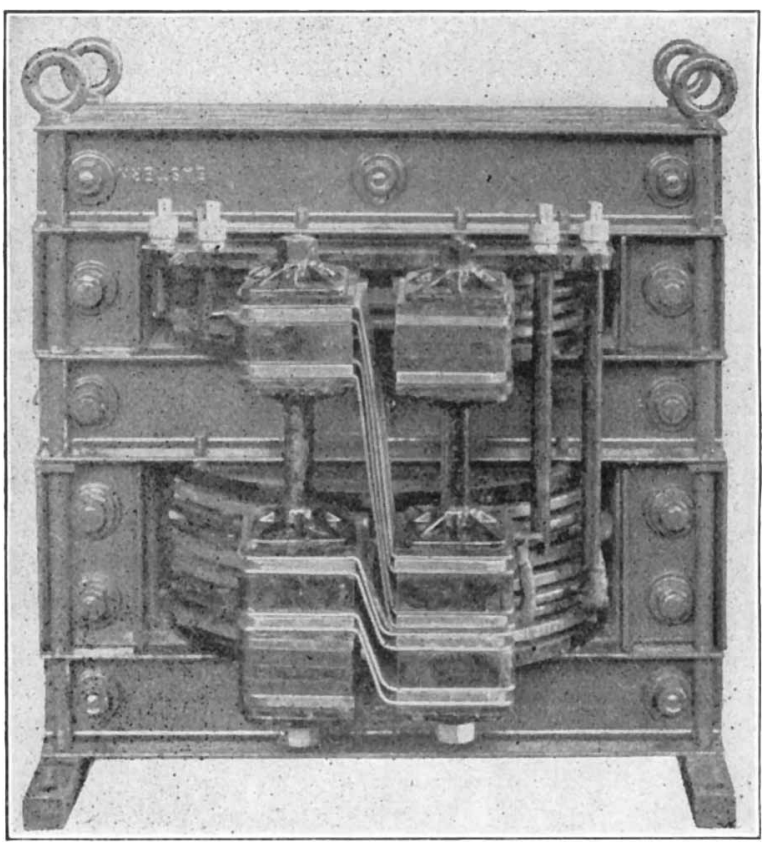

Fig. 4-Special Iron-Core Reactor with Auxiliary ShortCircuiting Winding

iron core of magnetic structure very similar to a transformer core, except that it usually does not form an ab- 
solutely closed magnetic circuit, but has a considerable air gap. The air gap improves the operating characteristics of the reactor, helping to reduce the magnetic saturation of the core through the current range for which the reactor is designed. This type of reactor may be air or oil insulated, and is usually self-cooled.

In the design of iron-core reactors which are depended upon to limit the current in a circuit, care must be exercised to insure that the iron core will not be over saturated at the maximum value of current which can

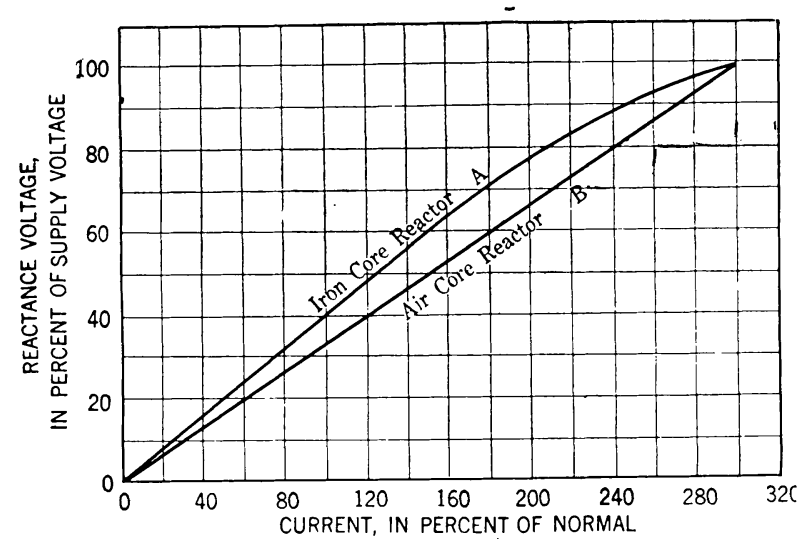

Fig. 5-Characteristic Curves of Iron- and Air-Core REACTORS

be forced through the circuit. Otherwise, the effective reactance of the coil will decrease greatly just when its full effect is most needed. A characteristic curve for an iron-core reactor is shown in Fig. 5 curve A. While this coil has a reactance voltage of 40.5 per cent at normal current, it gives only 100 per cent reactance voltage at 300 per cent normal current. That is, while the current has increased to three times normal, the reactance voltage has increased to only 2.47 times normal. Obviously, for current-limiting purposes the iron-core reactor should be designed so that its entire working range will fall below the "knee" of the curve.

The effect of the hysteresis in the iron core is to cause a distortion of the wave of the reactance voltage which is practically equivalent to a time-lag, thereby reducing the effectiveness of the reactor in maintaining the arc as the supply voltage approaches the zero value of its cycle.

The iron-core reactor is chiefly used in circuits carrying currents of less than about 50 amperes, when a reactance of more than 4 or 5 per cent is required. Under such conditions an air-core reactor would require a very large number of turns to give the desired reactance, and the iron-core design is considerably more economical.

A rather unusual iron-core reactor is illustrated in Fig. 4. This particular coil was designed for an installation in which two separate arcs were to be supplied from two separate low-voltage coils on a single transformer. Consequently it was necessary to place the reactor in the low-voltage circuit, the normal current in which was 4000 amperes. Furthermore, it was desired to be able to cut the reactor in and out of the circuit by push button control. As electrically operated circuit breakers of the current capacity required were quite expensive, an auxiliary coil of a large number of turns was wound on the reactor core. Short-circuiting this auxiliary coil had the same effect in the main circuit as short-circuiting the reactor; owing to the turn ratio, the current flowing in the auxiliary coil, when shortcircuited, was less than 200 amperes, and an inexpensive contactor could be used.

\section{AIR-CoRE REACTORS}

Air-core reactors, as the name implies, are coils with no iron or other magnetic material in their flux paths. Two typical methods of instruction of air-core reactors are illustrated. Fig. 6 shows what is termed the "diskcoil" type. This construction is used when a large number of turns are required to give the desired reactance, as in a low-current high-voltage circuit. A number of flat disk-coils of insulated conductors are assembled in a vertical stack and clamped between alloy spiders by means of insulators. The coils are spaced from each other by porcelain insulators, which support them at an angle of about 30 degrees to the horizontal, thus allowing good ventilation and cooling.

A "cast-in-concrete" reactor is illustrated in Fig. 7. In this reactor the conductor, of bare copper cable either solid or stranded, is solidly imbedded in specially

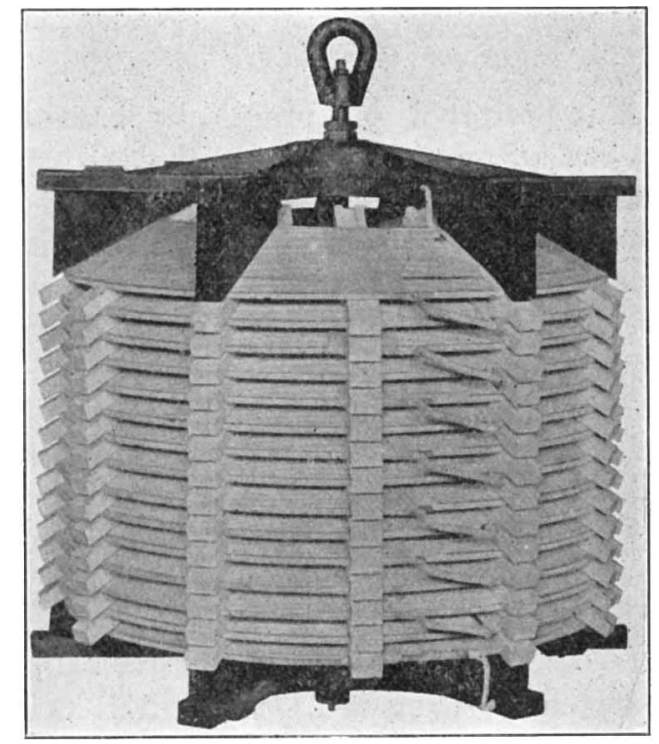

Fig. 6-Air-Core Reactor, Disk-Coll Type, Before ImPREGNATION OF COILS

treated concrete supports. The concrete is actually cast in place about the conductor, and is then cured in high-pressure steam, a process which secures a mechanical and electrical strength obtainable in no other way. The conductor and concrete are in very intimate mechanical contact, forming a strong rigid unit.

The vertical supporting members are mounted on a reinforced concrete base, which is in turn supported on 
porcelain posts when the circuit voltage is above 1000 .

The layers of conductors lie at an angle to the horizontal, alternate layers being dished in opposite directions, so that the space between conductors in adjacent layers increases with the number of included turns, and consequently the voltage, between the conductors.

There is no organic material whatever used in the construction of the cast-in-concrete reactor, conse-

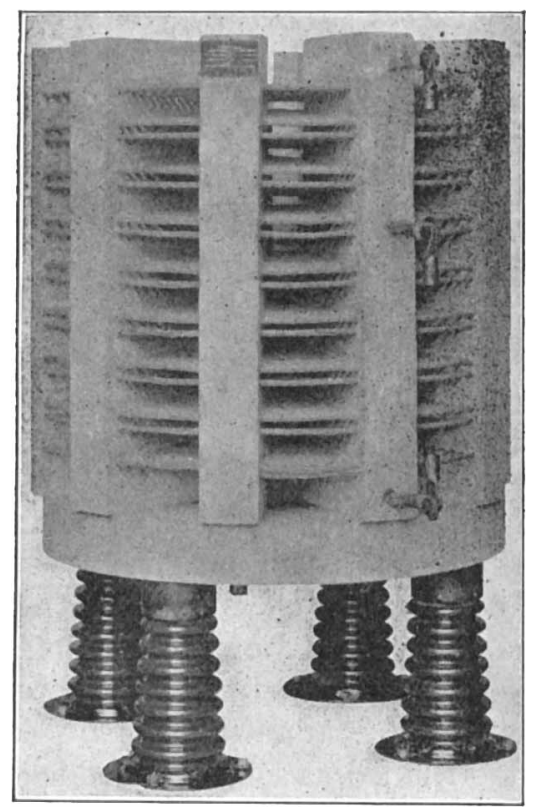

Fig. 7-Air-Core Reactor, Cast-in Concrete Type

quently it is fire-proof and practically indestructible, except by actual melting of the conductor.

The method of construction of the cast-in-concrete reactor does not lend itself readily to a design involving a very large number of turns. Consequently for circuits carrying very low currents the disk-coil air-core or the iron-core reactor is more economical.

There being no iron present in the magnetic circuit of the air-core reactor, its characteristic curve is a straight line; that is, its reactance voltage increases in exact proportion to the current, regardless of the value of the same. Furthermore, as there is no hysteresis, the flux does not lag behind the current but is always exactly proportional to it, and the full value of the reactive effect is realized.

\section{Position of REACTOR IN Circuit}

Reactors may be connected in either the high-tension supply circuit or between the transformer and furnace. Usually, if the current in the low-voltage circuit is of the order of 1500 amperes or higher, it is more economical, considering the cost of reactors, switches and connections, to put the reactors in the high-voltage circuit. This will also depend; however, on the voltage of the circuit and the amount of reactance required. No absolutely general rule can be given.

\section{Conclusions}

When reactance in addition to the normal value is required in an electric furnace circuit it may be obtained by increasing the reactance of the transformer, by suitably arranging the conductors, or by inserting reactance coils. The amount of reactance that can be obtained by the first two methods is limited, and they should only be used when the reactance is desired cotinuously in the circuit.

When varying values of reactance are required during different portions of the operating cycle, reactance
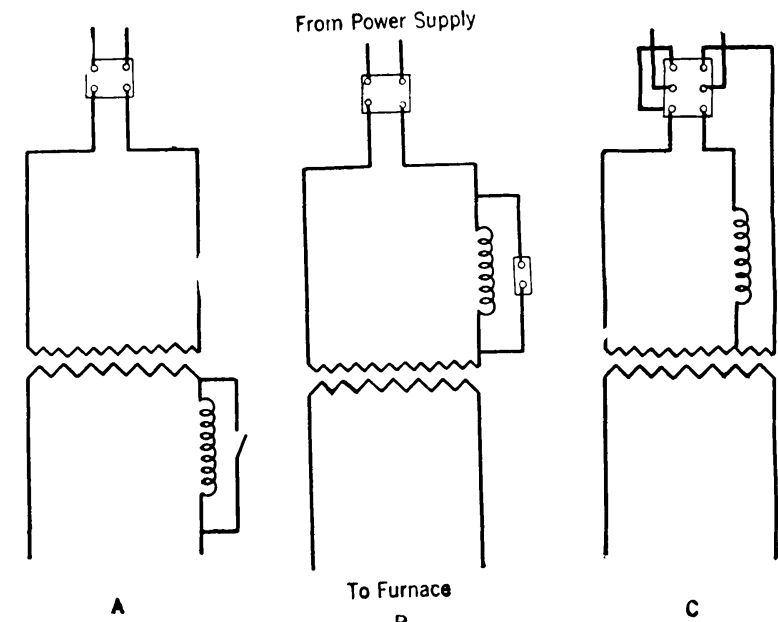

Fig. 8-Various Arrangements of Reactors in Electric Fornace Circuits

coils should be utilized. As the reactance is desired principally as a current limit, air-core reactors should be used wherever possible. Obviously, it would be advantageous if the reactance of the coil increased for current values above normal, as it would then be possible to have a fairly high power factor at normal load and still limit the maximum short-circuit current to a low value. The reactance voltage of the air-core reactor increases in direct proportion to the current flowing, while that of the iron-core type increases at a lower rate than the current. Obviously, the air-core reactor is preferable for furnace work.

In view of the considerations stated above, the castin-concrete air-core reactor, being substantial, efficient, and fire-proof, is in the opinion of the writer, the most suitable reactor for electric furnace circuits.

\section{A NEW FUEL FLUID}

Jerome Alexander, in a paper read before the American Chemical Society at St. Louis, April 15, announced the discovery of a new fuel fluid, greater in heat value than either coal or present fuel oils. He asserted that the fluid would prove valuable to navigation, permitting a wider cruising radius, and could be used for smoke screens. The new fuel utilizes coal waste and cheap tars, mixed by a secret process. 